\title{
Changing trend in clinico-mycological profile of dermatophytosis of skin in Eastern India
}

\author{
Reena Ray Ghosh ${ }^{1, *}$, Samatirtha Chandra ${ }^{2}$, Chandrika Goswami ${ }^{3}$, Mitali Chatterjee ${ }^{4}$ \\ ${ }^{\mathbf{1}}$ Associate Professor, ${ }^{2}$ Chief Medical Officer, ${ }^{3}$ Consultant Microbiologist, ${ }^{4}$ Professor \& HOD, ${ }^{1,4}$ Dept. of Microbiology, R. G. \\ Kar Medical College \& Hospital, Kolkata, West Bengal, ${ }^{2}$ ESIC Medical College, Kolkata ${ }_{s}^{3}$ M R Bangur Hospital, Kolkata, West \\ Bengal, India
}

*Corresponding Author:

Email: ghoshreena@hotmail.com

\begin{abstract}
Introduction: Dermatophytosis is one of the most prevalent cutaneous mycosis and a public health problem in West Bengal as well as in India. Its footprint spans far and wide across urban and rural Bengal, with a distribution that is determined by a multitude of factors like geography, environmental conditions, hygiene and sanitation. Despite its surging incidence, Dermatophytosis in Bengal remains a seldom studied topic.

Aim: The present study was undertaken to assess the burden of dermatophytosis by clinico-mycological evaluation using standard laboratory protocol and its correlation with clinical parameters.

Material and Methods: A cross sectional and observational study was conducted in 100 clinically diagnosed patients of dermatophytosis of skin attending the dermatology outpatient department of our tertiary care hospital. Skin scrapings, were collected from the active margins of the obvious new lesions and processed in the Mycology laboratory following standard protocol. All specimens were screened for presence of dermatophytes by direct microscopy using KOH DMSO preparation and were confirmed by fungal culture.

Results: Among all clinical types, Tinea corporis (58.0\%) was the predominant clinical condition. Males were affected more $(60.0 \%)$ than females. The predominantly affected age group was found to be $21-30$ years (35.0\%) followed by 31-40 years (24.0\%). Fungi were demonstrated in $72.0 \%$ cases by direct microscopy in $\mathrm{KOH}$ mount while $51.0 \%$ cases were found as culture positive. However $02.0 \%$ cases were KOH negative but culture positive. Microsporum audouinii (20.0\%) was the predominant species identified followed by Trichophyton rubrum (10\%). Some rare species like Microsporum persicolor, Microsporum distortum, Microsporum fulvum were also isolated in our study which are rarely documented in the literature till date.

Conclusions: In our study, males in the age group 21-40 years were most commonly affected. Tinea corporis was found to be the most common clinical presentation likely due to the poor hygiene and lack of sanitation amongst the study population, most of whom were manual labourers. Microsporum audouinii was the most common isolate.
\end{abstract}

Keywords: Dermatophyte, Dermatophytosis, Tinea, Microsporum audouinii.

\section{Introduction}

Dermatophytes are a set of fungi with capability to invade and infect keratinized tissue such as hair, nail and skin. Infection is generally cutaneous and restricted to the nonliving cornified layers because of the inability of the fungi to penetrate the deeper tissues or organs of immunocompetent hosts. ${ }^{1,2}$ Reactions to dermatophyte infection may range from mild to severe as a consequence of the host's reactions to the metabolic products of the fungus, the virulence of the infecting strain or species, the anatomic location of the infection, and local environmental factors.

The etiologic agents of the dermatophytoses are classified in three anamorphic (asexual or imperfect) genera, Epidermophyton, Microsporum, and Trichophyton, of anamorphic class Hyphomycetes of the Deuteromycota (Fungi Imperfecti). ${ }^{3}$ The descriptions of the genera essentially follow the classification scheme of Emmons on the bases of conidial morphology and formation of conidia. ${ }^{4}$

Traditionally infections caused by dermatophytes (ringworm) have been named according to the anatomical locations involved, by appending the Latin term designating the body site after the word Tinea. ${ }^{5,6}$
Several anatomic sites may be infected by a single dermatophyte species, and different species may produce clinically identical lesions.

Dermatophytosis is a major public health problem in the world today. Dermatophytosis has higher prevalence in the tropics because of high humidity, higher population density and poor sanitation and may even reach epidemic proportions. ${ }^{7}$ The disease is more frequent among men than woman. Several factors have been implicated to increase in disease such as trauma, increased sweating and diabetes. ${ }^{8}$ Despite increasing reports of dermatophytoses in different tropical and subtropical countries, there is scanty data on this issue from India especially from West Bengal which is situated in the Eastern part of India. In the current study, we have undertaken a clinico-mycological approach, correlating various demographic data such as age, and sex with identification of the fungus using standard techniques. 3,5 As the dermatophytic skin infections are more frequent when compared to those of hair and nails the study was confined to skin infections alone. The present study was undertaken with the objectives include to determine the incidence, contributing factors associated with dermatophytoses 
and occupational consequences related to disease. Also to isolate and characterise the causative dermatophytes and the species prevalent in this part of country.

\section{Material and Methods}

This is a cross-sectional and observational study over a period of one year from October 2014 to September 2015 conducted at R. G. Kar Medical College \& Hospital, Kolkata in West Bengal. The study population comprised of 100 clinically suspected cases of dermatophytoses attending Dermatology outpatients department at R. G. Kar Medical College \& Hospital, Kolkata during a period of one year. Our hospital which is a tertiary care hospital caters patients from densely populated metro city of Kolkata along with a wide suburban area surrounding it. Majority of the people are from rural areas with low socioeconomic background and poor literacy rate. Detailed history of onset of disease, duration of symptoms, trauma, occupation, drugs, associated co morbid conditions, family and personal history was taken. Enquiries were also made as to exposure to animals, cases or any other suspected sources. Collection and processing of the sample was done following standard laboratory procedures. Samples were collected from affected lesions. Whenever the patients presented with lesions at clinically different sites samples were collected from all those sites and each of these were processed and examined individually.

\section{Collection of sample}

Dermatophytes, as filamentous fungi, undergo radial growth. The central clearing of the classical annular lesion yields old poorly viable specimen. If the annular lesion is well defined, collection is best made from the advancing edges of the ring. Disinfection is carried out with $70 \%$ alcohol in gauze and the lesion is then scraped from centre outwards, crossing the annular margin, using a sterile scalpel blade. If the lesions have vesicles or bullae, the tops of the vesicles or bullae are clipped and included in the sample. Suppurating lesions are sampled with a swab when it is impractical to obtain scrapings. Other skin dermatophytoses, such as tinea pedis and tinea manuum, are scraped in such a way that the whole infected area is represented, since an advancing margin is often not evident .Sample materials are best transported in dry, strong dark paper folded in the manner of a herbarium packet. Dark paper allows easy visualization of small skin squames.

\section{Microscopic Examination}

Direct microscopy, although false negative in 5 to $15 \%$ of cases in ordinary practice, is a highly efficient screening technique. Scrapings of skin were subjected to $10 \% \mathrm{KOH}$ solution. ${ }^{1,3}$ The preparation was kept at room temperature for 30 mins. Subsequently examination was done under low power objective (10x) of the microscope for branching and septate hyphae and confirmation was made by high power objective (40x) of it.

\section{Culture}

Samples were inoculated after reducing the size of the samples to approximately to $1 \mathrm{~mm}$ as it was mentioned earlier. Inoculations were done at four (4) sites at well spaced interval onto Sabouraud's dextrose agar slants with chloramphenicol $(0.05 \mathrm{mg} / \mathrm{ml})$ and cyclohexamide $(0.5 \mathrm{mg} / \mathrm{ml}) .{ }^{1,3,6,9}$ Chloramphenicol was added to inhibit the growth of bacteria and cyclohexamide was used to inhibit the growth of saprophytic non-dermatophytic fungi. Inoculations of specimens were also done on DTM slopes for isolating dermatophytes where mixed pathogens were suspected. The tubes were incubated in BOD incubator at $28^{\circ} \mathrm{C}$ and also at room temperature to achieve good growth of some dermatophytes which prefer a little higher temperature. Regular examination of the tubes for detection and monitoring of fungal growth, were carried out. Tubes without any growth were discarded at 6 weeks. ${ }^{10}$ Growth on SDCA or SDCCA was examined for colony morphology, texture, pigmentation on surfaces (obverse and reverse).

Microscopic examination of colony was done by doing a lactophenol cotton blue mount to examine the hyphal structure, different vegetative structures formed by hyphal modifications, various reproductive structures like microconidia, macroconidia and chlamydoconidia. Urea hydrolysis was used to distinguish some species of Trichophyton and Microsporum. Hair perforation test was also performed as an additional aid to confirm final identification of the isolates.

\section{Results}

100 patients with dermatophytic skin infection were further clinically diagnosed as tinea corporis $(58.0 \%)$, tinea cruris $(6 \%)$, combined tinea corporis and cruris $(24 \%)$, tinea mannum(4\%), tinea faciei $(5 \%)$, tinea pedis $(3.0 \%)$. Male members were affected more than the female $(60.0 \%)$ and the M: F ratio was $3: 2$ [Table 1].

Clinical manifestation in relation to age showed the dermatophytic fungal infection was predominantly found in 21-30 years of age (35.0\%) followed by 31-40 years $(23.0 \%)$ as shown in Table 1.

Microscopic observation revealed presence of fungal hyphae by $\mathrm{KOH}$ mount in $72.0 \%$ cases as shown in Table 3. Total of $51.0 \%$ cases were positive in culture for dermatophytes, $02.0 \%$ cases which were $\mathrm{KOH}$ negative revealed positive culture.

Table 4 shows the distribution different sample in relation to clinical manifestation and age.

Table 5 shows identification of dermatophytic fungi. Among all the pathogens identified as Microsporum auoidinii (20\%) was the predominant species followed by Trichophyton rubrum (10\%) and 
Trichophyton tonsurans. Some rare species like Microsporum persicolor. Microsporum distortum,

Microsporum fulvum were isolated in our study.

Table 1: Dermatophytosis in relation to age and sex

\begin{tabular}{|l|c|c|c|}
\hline Age(years) & Male & Female & Total \\
\hline $11-20$ & 11 & 7 & 18 \\
\hline $21-30$ & 22 & 13 & 35 \\
\hline $31-40$ & 11 & 12 & 23 \\
\hline $41-50$ & 8 & 4 & 12 \\
\hline $51-60$ & 5 & 2 & 7 \\
\hline $61-70$ & 2 & 1 & 3 \\
\hline$>71$ & 1 & 1 & 2 \\
\hline Total & 60 & 40 & 100 \\
\hline
\end{tabular}

Table 2: Clinical types of dermatophytosis

\begin{tabular}{|l|r|r|}
\hline \multicolumn{1}{|c|}{ Clinical type } & No of isolates & Percentage \\
\hline Tinea corporis & & \\
\hline Tinea cruris & 58 & 58 \\
\hline Tinea coporis+T.cruris & 6 & 6 \\
\hline Tinea pedis & 24 & 24 \\
\hline Tinea faciei & 4 & 4 \\
\hline Tinea mannum & 5 & 5 \\
\hline & 100 & 3 \\
\hline
\end{tabular}

Table 3: Results obtained in direct microscopy $(\mathrm{KOH})$ and culture

\begin{tabular}{|l|c|c|c|}
\hline \multicolumn{1}{|c|}{ Culture } & KOH positive & KOH negative & Total \\
\hline Culture positive & 49 & 2 & 51 \\
\hline Culture negative & 23 & 26 & 49 \\
\hline Total & 72 & 28 & 100 \\
\hline
\end{tabular}

Table 4: Distribution of samples in relation to clinical manifestation and age

\begin{tabular}{|c|c|c|c|c|c|c|c|c|}
\hline Clinical & \multirow{2}{*}{\multicolumn{2}{|c|}{$\begin{array}{c}\text { Total no. } \\
\text { of samples } \\
\text { n }(\%)\end{array}$}} & $11-20$ & 21-30 & $31-40$ & $41-50$ & $51-60$ & $>61$ \\
\hline manifestation & & & n (\%) & n (\%) & n (\%) & n (\%) & n (\%) & $\mathrm{n}(\%)$ \\
\hline Tinea corporis & 58 & $(58.0)$ & 12 & 22 & 12 & 6 & 4 & 2 \\
\hline Tinea cruris & \multicolumn{2}{|c|}{$6(6.0)$} & 2 & 1 & 1 & 1 & 1 & \\
\hline $\begin{array}{l}\text { Tinea corporis+ } \\
\text { Tinea cruris }\end{array}$ & \multicolumn{2}{|c|}{$24(24.0)$} & 2 & 9 & 8 & 1 & 2 & 2 \\
\hline Tinea manuum & \multicolumn{2}{|c|}{$3(3.0)$} & 0 & 1 & 0 & 1 & 0 & 1 \\
\hline Tinea faciei & \multicolumn{2}{|c|}{$5(5.0)$} & 1 & 1 & 1 & 2 & 0 & 0 \\
\hline Tinea pedis & \multicolumn{2}{|c|}{$4(4.0)$} & 1 & 1 & 1 & 1 & 0 & 0 \\
\hline Total & 100 & $(100)$ & $18(18.0)$ & $35(35.0)$ & $23(23.0)$ & $12(12.0)$ & $7(7.0)$ & $5(5.0)$ \\
\hline
\end{tabular}

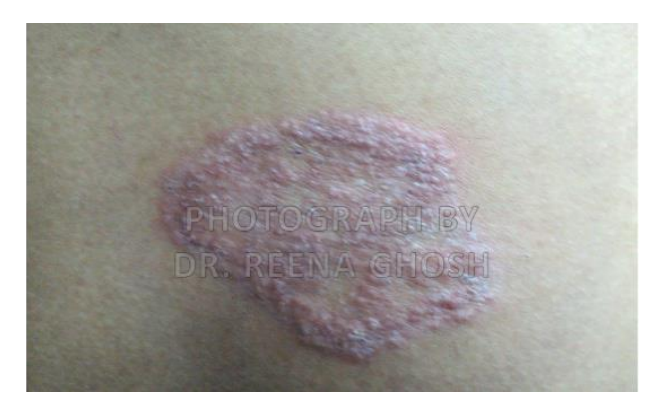

Fig. 1: Tinea corporis. Psoriasiform lesion. Silvery scales covered this infiltrated maculopapular area. Some vesicles are evident in several places on the surface. The periphery was quite erythematous and inflammed 


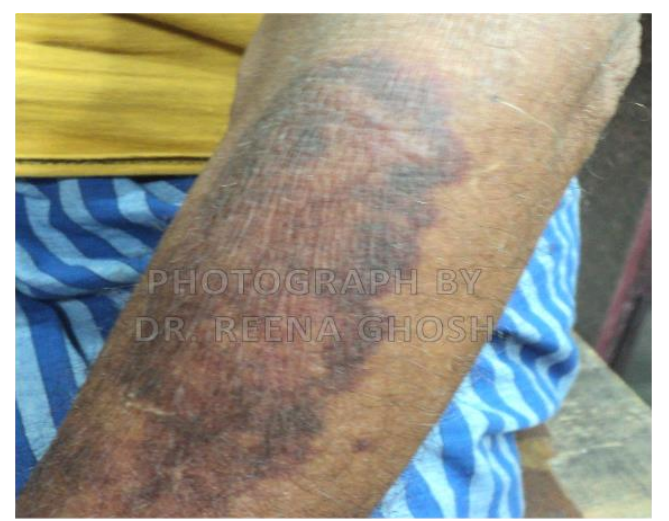

Fig. 2: Tinea corporis. Plaque like lesion. The outer ring is infiltrated and has a red rolled border with a few vesicles. The remaining surface of the lesion is smooth, firm, infiltrated and plaque like

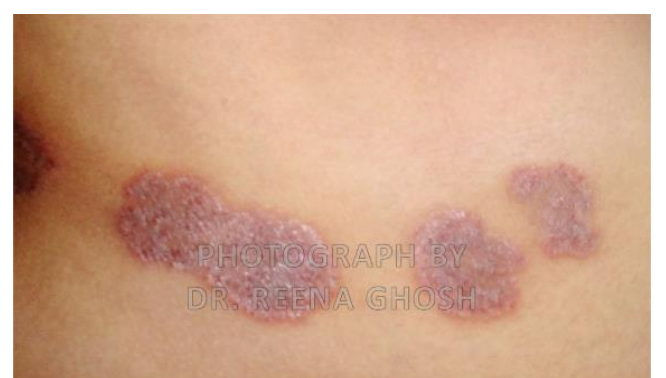

Fig. 3: Tinea corporis on right lateral aspect of abdomen. Lesions are raised and erythematous caused by Trichophyton mentagrophyte var quinkeanum

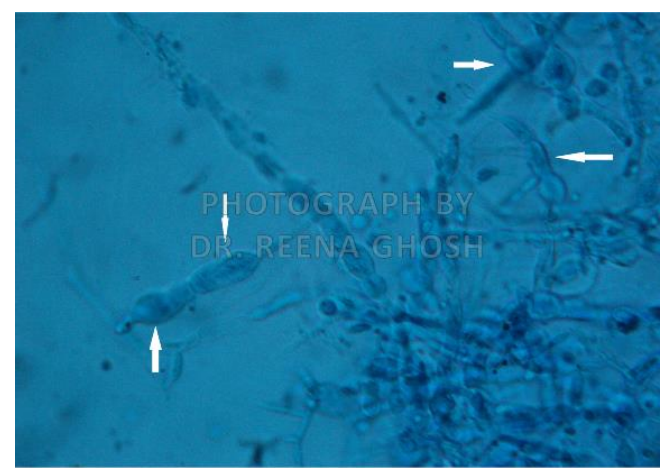

Fig. 4: M.audouinii, irregular crooked tip macroconidia(white arrow) are characteristic feature (LPCB mount X800)
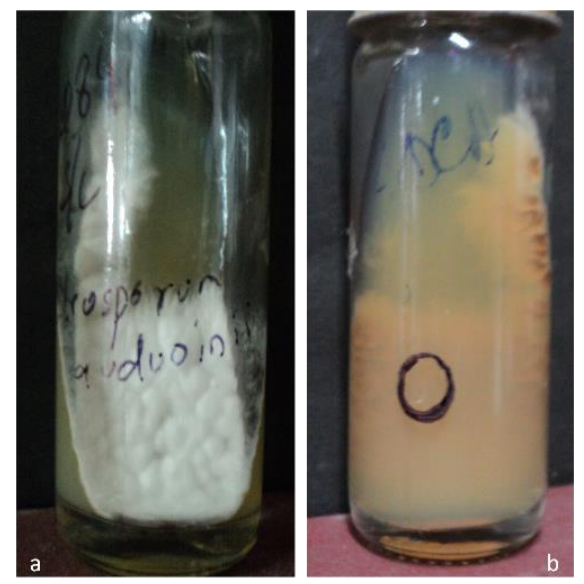

Fig. 5: Colony morphology of Microsporum audouinii grown on SDCA, a) obverse b) reverse 
Table 5: Prevalence pattern of species of dermatophytes

\begin{tabular}{|c|c|c|c|c|c|c|c|c|c|c|c|c|c|}
\hline $\begin{array}{c}\text { Clinical } \\
\text { manifestation }\end{array}$ & 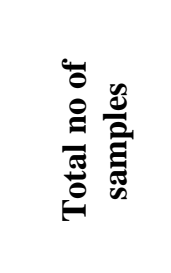 & 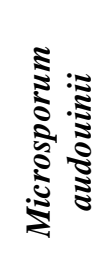 & 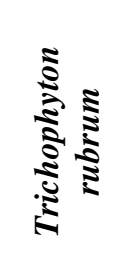 & 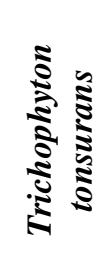 & 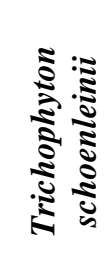 & 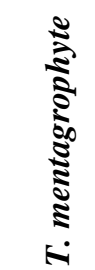 & 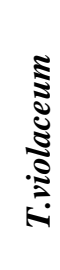 & 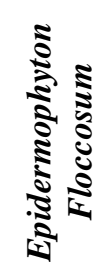 & 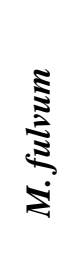 & 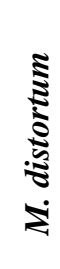 & 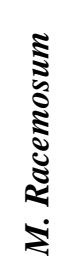 & 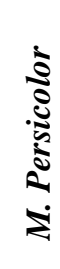 & : \\
\hline & $\mathrm{n}(\%)$ & $\mathrm{n}(\%)$ & $\mathrm{n}(\%)$ & $\mathrm{n}(\%)$ & $\mathrm{n}(\%)$ & $\mathrm{n}(\%)$ & & $\mathrm{n}(\%)$ & & & & & \\
\hline Tinea corporis & $58(25.0)$ & 12 & 06 & 05 & 03 & 02 & 01 & 01 & 01 & 01 & 01 & 01 & 34 \\
\hline Tinea cruris & $6(5.0)$ & 01 & 01 & 00 & 01 & & & 00 & & & & & 03 \\
\hline $\begin{array}{l}\text { Tinea corporis } \\
+ \text { Tinea cruris }\end{array}$ & $24(24.0)$ & 06 & 02 & 01 & 00 & & 01 & 00 & & & & & 10 \\
\hline Tinea manuum & $3(3.0)$ & 00 & 00 & 00 & 00 & & & 01 & & & & & 01 \\
\hline Tinea pedis & $4(4.0)$ & 00 & 01 & 00 & 00 & & & 00 & & & & & 01 \\
\hline Tinea faciei & $5(5.0)$ & 01 & 00 & 00 & 01 & & & 00 & & & & & 02 \\
\hline Total & $100(100.0)$ & $0(20)$ & $10(10)$ & $6(6.0)$ & $5(5.0)$ & 02 & 02 & 02 & 01 & 01 & 01 & 01 & 51 \\
\hline
\end{tabular}




\section{Discussion}

Dermatophytosis has a wide geographical distribution; the species of dermatophyte causing infection may vary from region to region and are geographically restricted except some species like Trichophyton rubrum which have a cosmopolitan distribution. ${ }^{5}$ The present study was conducted to assess the clinical and culture profile of dermatophytic skin infection.

In the present study out of 100 samples processed $60 \%$ were from males and $40 \%$ were from female patients. Study showed that males were predominantly affected than females. ${ }^{2,9,11,12}$ Male preponderance may be correlated with the occupational hazards related to their nature of work, the frequent interaction with different people of the society, environmental conditions such as hot and humid weather, poor personal hygiene and illiteracy are other major factors that influence dermatophytosis.

In our study of 100 cases of tinea, tinea corporis was the commonest clinical type observed (58.0\%) followed by mixed infection of tinea corporis \& cruris $(24.0 \%)$ being the second commonest type [Table 2].Tinea corporis as predominant clinical type is in line with the different studies in West Bengal, Kasmir, Manipal, Assam and other studies., ${ }^{83-16,19}$ Tinea corporis had been reported to be the most common clinical type even in few other countries like Spain and Brazil. ${ }^{17,18}$ Verma et $a l^{19}$ and Sardari et $a l^{20}$ found tinea cruris as the commonest clinical type which is in contrast to our study. Among mixed clinical types, tinea corporis with tinea cruris was highest (24 cases, $73.91 \%$ ). Similar findings have been reported by Peerapur et al. $^{12}$

Majority $(35.0 \%)$ of the infection has occurred between 21-30 years followed by age group of 31-40 years (23\%) [Table1]. The majority affected in our study were from the age group of 21-40 probably as they were employed as manual labourers and daily wage earners; due to the lower hygiene and sanitation facilities available to them. This agrees with results from other studies. ${ }^{2,7,15,20-23}$

As in our study, fungal infection in clinical isolates was detected by $\mathrm{KOH}$ mount which later on confirmed by culture as shown in [Table 3]. Positivity rates of $\mathrm{KOH}$ mount was found to be $72.0 \%$. Of which $49.0 \%$ of culture was positive for dermatophytes and remaining $02.0 \%$ of culture positive were $\mathrm{KOH}$ negative .This study is in accordance with the study of Doddamani PV et $\mathrm{al}^{9}$ who reported $65.0 \% \mathrm{KOH}$ positive and $39.0 \%$ culture positive. $9.0 \%$ were $\mathrm{KOH}$ negative and culture positive. Singh $\mathrm{S}$ et $\mathrm{al}^{2}$ also found more positivity rate of detection by $\mathrm{KOH}$ mount $(60.4 \%)$ than culture $(44.6 \%)$ and only $3.8 \%$ were $\mathrm{KOH}$ negative and culture positive. Direct microscopic examination using $\mathrm{KOH}$ is very simple technique giving rapid presumptive diagnosis.
Distribution of dermatophytic fungi in different clinical patterns showed Microsporum audouinii (20\%) was the commonest aetiological agent followed by Trichophyton rubrum $(10 \%)$ which is contrast to most of the earlier studies, $2,6,7,16,22,23,25-27$ where Trichophyton rubrum was the commonest isolate like Bindu $\mathrm{V}$ et al ${ }^{22}$ in $2002(66.2 \%)$, Sumana V et $\mathrm{al}^{27}$ in $2004(60 \%)$, Peerapur B V et $\mathrm{al}^{16}$ in 2004 (43.7\%). In a study by Grover et al ${ }^{28}$ Trichophyton tonsurans was commonest isolate followed by Trichophyton rubrum. Although Microsporum audouinii was the most frequent established etiology of tinea capitis, in our study, none of them were isolated from tinea capitis as there was no such case of dual lesions causing clinical condition of 'tinea capitis' and 'tinea corporis' in the same patient .

Brash J et al $2015^{29}$ found M.audouinii as isolate from tinea corporis in a German boy who had a history of animal exposure.

Antifungal drug resistance is becoming a common problem in patients and is inevitable due to wide availability and use of drugs. Establishment of a reproducible method of susceptibility testing will be important components of a strategy to limit the emergence of resistance to these agents.

\section{Conclusion}

The study shows Dermatophytosis is prevanlant across all age groups even in Bengal. The most common clinical presentation was Tinea Corporis. Infection was predominant in males. The age group with highest prevalence was 21-40 years. The most common fungi isolated was Microsporum audouinii followed by Trichophyton rubrum. The study also concurred that diagnosis of Dermatophytosis clinically followed by $\mathrm{KOH}$ examination was the best strategy and Culture was the gold standard confirmatory test. We reiterate that even if culture facility not available, $\mathrm{KOH}$ examination should be performed on routine basis. Antifungal therapy selection should be based on the identity of the causative agent to ensure efficacy and safety.

\section{References}

1. Larone DH. Dermatophytes. In: medically important fungi - A guide to identification. $4^{\text {th }}$ Edn; ASM Press, Washington DC 2002;241-53.

2. Beena P M, Singh S, Profile of dermatophyte infectins in Baroda, Ind J Derm Venereal Leprol 2003;69:281-83.

3. Weitzman I, Summerbell RC. The dermatophytes. Clin Microbiol Rev 1995;8(2):240-59.

4. Emmons, C. W. 1934. Dermatophytes: natural groupings based on the form of the spores and accessory organs. Arch Dermatol Syphilol 30:337-62.

5. Kannan P, Janaki C, Selvi G S, Prevalence of Dermatophytes and other fungal agents isolated from clinical samples, Ind J Med Microbial 2006;24:212-15.

6. KAK S, Bhat RM, Boloor R, Nandakishore B, Sukumar D. A Clinical and Mycological Study of Dermatophytic Infections. Ind J Dermatol 2014;59(3):262-67. 
7. Sentamilselvi G, Kamalam A, Ajithadas K, Janaki C, Thambiah AS. Scenario of chronic dermatophytosis: an Indian study. Mycopathologia 2017;140(3):129-35. Available from:

http://www.ncbi.nlm.nih.gov/pubmed/9691500

8. Das K, Basak S, Ray S. A study on superficial fungal infection from West Bengal. A brief report. J Life Sci 2009;1(1):51-5.

9. Doddamani P V, Harshan K H, Kanta R C, Gangane Sunil R, Isolation, Identification and Prevalence of Dermatophytes in Tertairy care Hospital in Gulbarga District, People's J Scientific Res 2013;6:2.

10. Sarkar M, Ray R, Halder P, Ghosh AP, Chatterjee M. Clinico - Mycological study of Onychomycosis -- A study in a tertiary care hospital in Kolkata. IOSR J Dent Med Sci 2016;15(9):78-83.

11. Balakumar S, Rajan S, Thirunalasundari T, Jeeva S. Epidemiology of dermatophytosis in and around Tiruchirapalli, Tamilnadu, India. Asian Pacific J Trop Dis 2017;2(4):286-89. Available from: http://linkinghub.elsevier.com/retrieve/pii/S22221808126 00620

12. Peerapur BV, Inamdar AC, Pushpa PV, Srikant B. Clinico Mycological Study of Dermatophytosis in Bijapur. Ind J Dermatol Venerol Leprol 2004;22:273-74.

13. Stephen S, Rao KNA. Superficial mycoses in Manipal, Ind J Dermatol Venereol Leprol 1975;41:106.

14. Phadke SN .Dermatophytosis in Jabalpur (Madhya Pradesh). Ind J Pathol Bacteriol 1973;16:42.

15. Bhardwaj G. Dermatophytoses in Kashmir (India). Mycosen 1987;30:135.

16. Sumit Kumar, P Shrikara Mallya, Pallavi Kumari. "Clinico-Mycological Study of Dermatophytosis in a Tertiary Care Hospital". Int J Scientific Study 2014;1(6):27-32.

17. Fortuno B, Torres L, Simil E, Seoane A, Uriel JA, Santacruz C. Dermatophytes isolated in our clinics, 5year study in Zaragoza. Enferm Infecc Microbiol Clin 1997;15:536-39.

18. Chinelli PA, Sofiatti Ade A, Nunes RS, Martins JE. Dermatophyte agents in the city of Sao Paulo, from 1992 to 2002.Rev Inst Med Trop Sao Paulo 2003;45:259-63.

19. SS Sen, ES Rasul. Dermatophytosis in Assam. Indian Journal of Medical Microbiology. 2006;24(1):77-8.
20. Sardari L, Sambhashiva RR, dandapani R. clinico mycological study of dermatophytes in a coastal area. Ind J Dermatol Venereol Leprol 1983;49:2:71-5.

21. Nita Patwardhan, Rashmika Dave et al. "Dermatomycosis in and around Aurangabad" Ind J Pathol Microbial 1999;42(4):455-62.

22. Seema Bhaduria, Neetu Jain et al. Dermatophytosis in Jaipur: study of incidence, clinical features and causal agents". Ind J Microbiol 2001;41:207-10.

23. Bindu V. Pavithran K. Clinico mycological study of dermatophytosis in Calicut. Ind J Dermatol Vernerol Leprol 2002;68(5):259-61.

24. Sumathi S, Mariraj J, Ramesh R, Krishna S, Clinico Mycological study of Dermatophytes. Int J Pharm Biomed Res 2013;4:132-34.

25. Verma BS, Vaishnav VP, Bhat RP. A study of dermatophytosis. Ind J Dermatol Venerol Leprol 1970;36:182

26. Venkatesan G, Singh AJAR, Murugesan AG, Janaki C, Shankar SG. Trichophyton rubrum-the predominant etiological agent in human dermatophytoses in Chennai India. Afr J Microbiol Res 2007;1(1):9-12.

27. V Sumana, MA Singaracharya. Dermatophytosis in Khammam. Ind J Microbiol 2004;47(2):287-89.

28. Grover SC, Roy PC. Clinicomycological profile of superficial mycosis in a hospital in North East India. Med J Armed Forces Ind 2003;59:114-69

29. Brasch J, Müller S, Gräser Y. Unusual strains of Microsporum audouinii causing tinea in Europe. Mycoses 2017;58(10):573-7. Available from: http://doi.wiley.com/10.1111/myc. 12358 . 